\title{
Optical characterization of WISE selected blazar candidates
}

\author{
Raniere de Menezes ${ }^{1,2}$, Harold A. Peña-Herazo ${ }^{1,3,4,5}$, Ezequiel J. Marchesini ${ }^{1,4,7,8,9}$, Raffaele D'Abrusco ${ }^{6}$, \\ Nicola Masetti ${ }^{9,10}$, Rodrigo Nemmen ${ }^{2}$, Francesco Massaro ${ }^{1,4,5,13}$, Federica Ricci ${ }^{11}$, Marco Landoni ${ }^{12}$, \\ Alessandro Paggi ${ }^{4,5}$, and Howard A. Smith ${ }^{6}$ \\ 1 Dipartimento di Fisica, Università degli Studi di Torino, Via Pietro Giuria 1, 10125 Torino, Italy \\ e-mail: raniere.m.menezes@gmail.com \\ 2 Universidade de São Paulo, Departamento de Astronomia, São Paulo, SP 05508-090, Brazil \\ 3 Instituto Nacional de Astrofísica, Óptica y Electrónica (INAOE), Apartado Postal 51-216, 72000 Puebla, Mexico \\ ${ }^{4}$ INFN - Istituto Nazionale di Fisica Nucleare, Sezione di Torino, Via Pietro Giuria 1, 10125 Turin, Italy \\ 5 INAF-Osservatorio Astrofisico di Torino, Via Osservatorio 20, 10025 Pino Torinese, Italy \\ ${ }^{6}$ Center for Astrophysics | Harvard and Smithsonian, 60 Garden Street, Cambridge, MA 20138, USA \\ 7 Facultad de Ciencias Astronómicas y Geofísicas, Universidad Nacional de La Plata, Paseo del Bosque, B1900FWA La Plata, \\ Argentina \\ 8 Instituto de Astrofísica de La Plata, CONICET-UNLP, CCT La Plata, Paseo del Bosque, B1900FWA La Plata, Argentina \\ 9 INAF - Osservatorio di Astrofisica e Scienza dello Spazio, Via Gobetti 93/3, 40129 Bologna, Italy \\ 10 Departamento de Ciencias Físicas, Universidad Andrés Bello, Fernández Concha 700, Las Condes, Santiago, Chile \\ 11 Instituto de Astrofísica and Centro de Astroingeniería, Facultad de Física, Pontificia Universidad Católica de Chile, Casilla 306, \\ Santiago 22, Chile \\ 12 INAF - Osservatorio Astronomico di Brera, Via E Bianchi 46, Merate, LC, Italy \\ 13 Consorzio Interuniversitario per la Fisica Spaziale (CIFS), Via Pietro Giuria 1, 10125 Torino, Italy
}

Received 27 June 2019 / Accepted 14 August 2019

\begin{abstract}
Context. Over the last decade more than five thousand $\gamma$-ray sources have been detected by the Large Area Telescope (LAT) onboard the Fermi Gamma-ray Space Telescope. Given the positional uncertainty of the telescope, nearly $30 \%$ of these sources remain without an obvious counterpart at lower energies. This has motivated the release of new catalogs of $\gamma$-ray counterpart candidates and several follow up campaigns in the last decade.

Aims. Recently, two new catalogs of blazar candidates were released. These are the improved and expanded version of the WISE Blazar-Like Radio-Loud Sources (WIBRaLS2) catalog and the Kernel Density Estimation selected candidate BL Lacs (KDEBLLACS) catalog, both selecting blazar-like sources based on their infrared colors from the Wide-field Infrared Survey Explorer (WISE). In this work we characterize these two catalogs, clarifying the true nature of their sources based on their optical spectra from SDSS data release 15 , thus testing their efficiency in selecting true blazars.

Methods. We first selected all WIBRaLS2 and KDEBLLACS sources with available optical spectra in the footprint of Sloan Digital Sky Survey data release 15 . We then analyzed these spectra to verify the nature of each selected candidate and to measure the fraction of the catalogs composed by spectroscopically confirmed blazars. Finally, we evaluated the impact of selection effects, especially those related to optical colors of WIBRaLS2/KDEBLLACS sources and their optical magnitude distributions.

Results. We found that at least $\sim 30 \%$ of each catalog is made up of confirmed blazars, with quasars being the major contaminants in the case of WIBRaLS2 $(\approx 58 \%)$ and normal galaxies in the case of KDEBLLACS $(\approx 38.2 \%)$. The spectral analysis also allowed us to identify the nature of 11 blazar candidates of uncertain type (BCUs) from the Fermi-LAT fourth Point Source Catalog (4FGL) and to find 25 new BL Lac objects.
\end{abstract}

Key words. BL Lacertae objects: general - catalogs - galaxies: active - radiation mechanisms: non-thermal

\section{Introduction}

One of the main challenges of modern $\gamma$-ray astronomy in the era of the Fermi Large Area Telescope (LAT) is the association of $\gamma$-ray sources with their low-energy counterparts (Massaro et al. 2015a, 2016a). The main problem underlying this challenge is the large positional uncertainty of $\gamma$-ray-detected sources, typically $\sim 4^{\prime}$ in the Fermi-LAT fourth Point Source Catalog ${ }^{1}$ (4FGL; Fermi-LAT Collaboration 2019). The association task also affects the source classification that covers up to

\footnotetext{
1 https://fermi.gsfc.nasa.gov/ssc/data/access/lat/8yr_ catalog/
}

$\sim 70 \%$ of the 4FGL leaving 1521 unidentified/unassociated $\gamma$-ray sources (UGSs) to date.

These UGSs are distributed uniformly throughout the whole sky - although showing some concentration towards the Galactic plane $\left(|b|<20^{\circ}\right)$ - indicating that most of them could have extragalactic nature. The $\gamma$-ray sky is mainly populated by nonthermal sources and in particular $\sim 80 \%$ of 4FGL associated sources are classified as blazars. These blazars belong to one of the rarest classes of active galactic nuclei (AGNs), whose emission arises from particles accelerated in a relativistic jet closely aligned with the line of sight (Blandford et al. 1978). It is therefore expected that a significant fraction of UGSs are blazars, at least at high Galactic latitudes. 
Blazars are divided into BL Lacs (BZBs) and blazars of quasar type labeled in the Roma-BZCAT as BZQs (Massaro et al. 2015b), and are classified based on their optical spectra, where the former have featureless optical spectra, or only absorption lines of Galactic origin and weak and narrow $(<5 \AA$; Stickel et al. 1991; Landoni et al. 2014, 2015a,b) emission lines; and the latter have flat radio spectra, with optical spectra showing broad emission lines and a dominant intrinsically blue continuum. Blazars, emitting nonthermal radiation over the whole electromagnetic spectrum, also show large variability at all wavelengths, a flat or inverted radio spectrum, significant polarized emission, and in some cases even apparent super-luminal motion (Urry \& Padovani 1995; Abdo et al. 2010).

Blazars occupy a specific region in the mid-infrared (MIR) color space defined by the Wide-field Infrared Survey Explorer filters (WISE; Wright et al. 2010). This region is known as the WISE Blazar Strip (Massaro et al. 2011, 2012a; D'Abrusco et al. 2012) when it is described in a two-dimensional space, and the locus when it is modeled in the full three-dimensional WISE color space (D'Abrusco et al. 2014). Such distinctive MIR colors are due to the nonthermal emission arising from their relativistic jets (Böttcher 2007). This discovery led to searches for blazar-like sources lying within the positional uncertainty ellipses of UGSs that could be their potential counterparts (D'Abrusco et al. 2014; Massaro et al. 2015a).

One of the catalogs that provides the largest number of associated blazar candidates for Fermi catalogs is the WISE BlazarLike Radio-Loud Sources catalog (WIBRaLS; D'Abrusco et al. 2014, 2019). Selected sources in the WIBRaLS are detected in all four MIR WISE bands (nominally at 3.4, 4.6, 12 and $22 \mu \mathrm{m}$ ) and show MIR colors similar to those of confirmed Fermi blazars. Sources in WIBRaLS are also required to have a radioloud counterpart (see more details in Sect. 2.1). In the latest release of the WIBRaLS catalog (hereinafter, WIBRaLS2), an additional list of candidate BZBs has been built based on a kernel density estimation (KDE) technique, namely KDEBLLACS (D'Abrusco et al. 2019). Sources in KDEBLLACS are also radio-loud. Sources in both catalogs are selected with Galactic latitude $|b|>10^{\circ}$.

The main goal of the present analysis is to characterize sources listed in both WIBRaLS2 and KDEBLLACS on the basis of the optical spectra available for those lying in the footprint of the Sloan Digital Sky Survey (SDSS; York et al. 2000) fifteenth data release (DR15; Aguado et al. 2019). We aim to classify them spectroscopically mainly to determine the source classes that contaminate the WIBRaLS2 selection criteria. SDSS is ideal for our purposes since it is the survey with the highest number of spectroscopic objects available in the literature.

The paper is organized as follows: in Sect. 2 we describe samples used to carry out our analysis, providing basic details of both WIBRaLS2 and KDEBLLACS, selecting those sources in the SDSS footprint. Sections 3 and 4 describe our spectral classification method and results, respectively. Finally, we summarize our results and conclusions in Sect. 5.

Throughout this work we use $h=0.70, \Omega_{\mathrm{m}}=0.30$, and $\Omega_{\Lambda}=0.70$, where the Hubble constant is $H_{0}=$ $100 \mathrm{hm} \mathrm{s}^{-1} \mathrm{Mpc}^{-1}$ (Tegmark et al. 2004). Spectral indices are defined by flux density $S_{v} \propto v^{-\alpha}$, indicating a flat spectrum when $\alpha<0.5$. The WISE magnitudes are in the Vega system and are not corrected for the Galactic extinction, since, as shown in D'Abrusco et al. (2014), such correction only affects the magnitude at $3.4 \mu \mathrm{m}$ for sources lying close to the Galactic plane and ranges between $2 \%$ and $5 \%$ of the magnitude, thus not significantly affecting the results. WISE bands are indicated

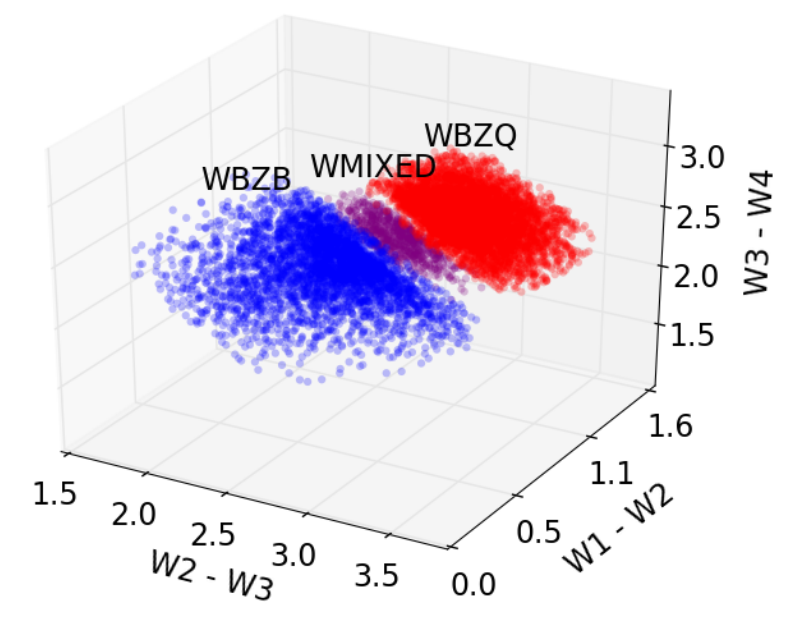

Fig. 1. Entire WIBRaLS2 sample divided into three categories according to the MIR colors of the sources: BZB candidates (WBZBs, in blue), BZQ candidates (WBZQs, in red), and blazars with intermediate colors (WMIXEDs, in purple).

as $W 1, W 1, W 3$, and $W 4$, and correspond respectively to the nominal wavelengths at $3.4,4.6,12$, and $22 \mu \mathrm{m}$.

\section{Sample selection}

For this analysis we used observations available in the AllWISE catalog (Cutri et al. 2013, 2014), which contains astrometry and photometry in the IR for $3 \times 10^{7}$ sources in $W 1, W 1, W 3$, and $W 4$ bands. The catalog includes data from the cryogenic and postcryogenic survey phases (Mainzer et al. 2011), increasing to $\sim 10^{8}$ sources detected only on the first three bands $W 1, W 1$, and $W 3$.

\subsection{WIBRaLS2}

The WIBRaLS2 catalog was conceived to provide a good sample of potential $\gamma$-ray sources based on IR and radio data. It includes MIR sources detected in the four WISE filters, with colors similar to those of blazars listed in the Fermi-LAT fouryear Point Source Catalog (3FGL; Acero et al. 2015), spatially cross-matched with a radio counterpart found in one of the three major radio surveys: the National Radio Astronomy Observatories Very Large Array (VLA) Sky Survey (NVSS; Condon et al. 1998), the VLA Faint Images of the Radio Sky at Twentycentimeter Survey (FIRST; White et al. 1997; Helfand et al. 2015), and the Sydney University Molonglo Sky Survey Source Catalog (SUMSS; Mauch et al. 2003); and selected to be radioloud based on their $q_{22}$ spectral parameter, defined as $q_{22}=$ $\log \left(S_{22 \mu \mathrm{m}} / S_{\text {radio }}\right)$, with $S_{22 \mu \mathrm{m}}$ and $S_{\text {radio }}$ being the flux densities at $22 \mu \mathrm{m}$ and in radio, respectively. The total number of blazar candidates in WIBRaLS2 is 9541 and they are classified (see Fig. 1) as candidate BZBs, candidate BZQs, or MIXEDs (hereafter referred to as WBZBs, WBZQs, and WMIXEDs, respectively), the latter being defined as those with MIR colors which are intermediate between WBZBs and WBZQs (for more details, see D'Abrusco et al. 2019).

In this paper, we have cross-matched the WIBRaLS2 catalog with SDSS DR15 associating blazar candidates listed therein with their optical counterparts within an angular separation of $2^{\prime \prime}$ (Massaro et al. 2014). We found a total number of 3407 unique associations and then used the SDSS Science Archive Server ${ }^{2}$ to select only those sources with an available optical spectrum.

2 https://dr15.sdss.org/optical/spectrum/search 


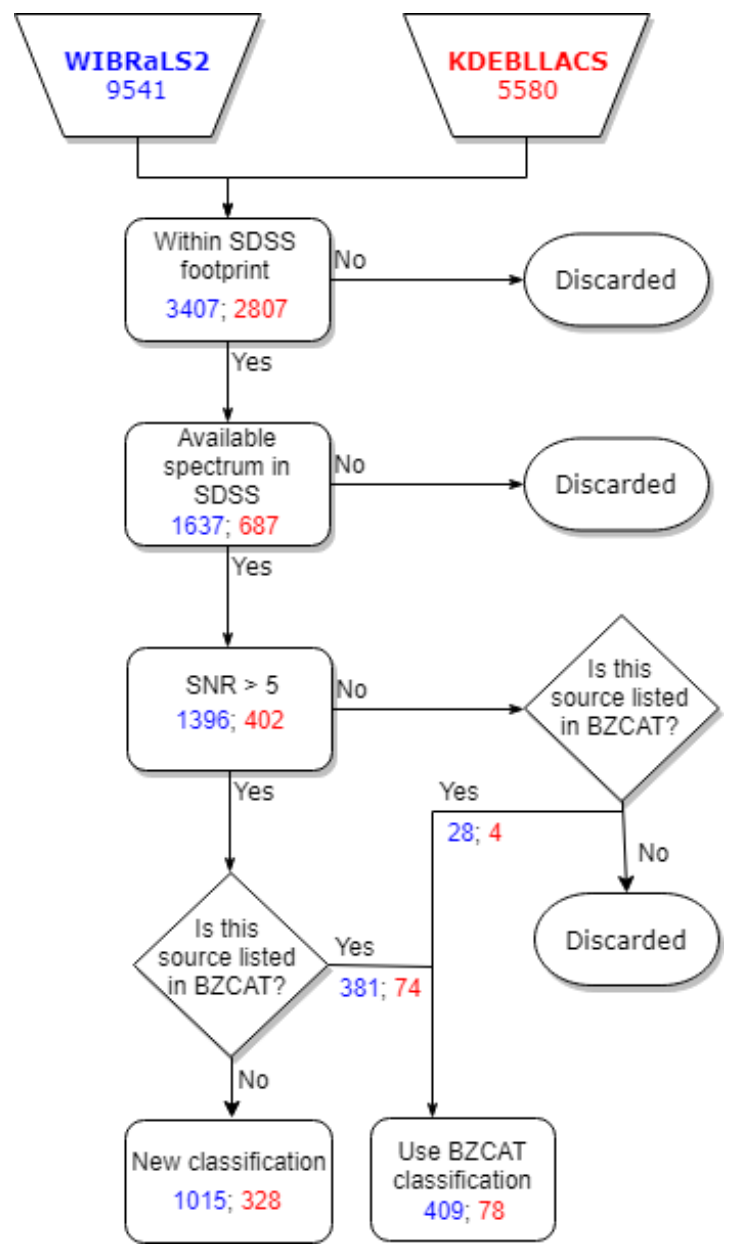

Fig. 2. Flow chart with all steps adopted in the sample selection and spectral classification. The numbers in blue refer to sources in WIBRaLS2 while numbers in red refer to sources in KDEBLLACS after each step.

We then selected only those sources with a spectral signal-tonoise ratio $(\mathrm{S} / \mathrm{N})$ above 5 and sources with $S / N<5$ but listed in Roma-BZCAT. This led us to a final sample of 1424 WIBRaLS2 sources, which corresponds to $\sim 15 \%$ of the whole WIBRaLS2. A flowchart describing the effects of our selection of the candidates in the WIBRaLS2 catalog is displayed in Fig. 2.

\subsection{KDEBLLACS}

The KDEBLLACS catalog was built by first applying a kernel density estimation (KDE) to a two-dimensional distribution of training set sources of BZB type in the WISE $W 1-W 2 \times W 2-W 3$ color diagram to determine its probability distribution function (PDF). Sources were then selected when located within the $90 \%$ isodensity contour of the training set (D'Abrusco et al. 2019) built with the KDE. The color uncertainty ellipses of each source in KDEBLLACS must be fully contained in this $90 \%$ contour. Sources in KDEBLLACS are radio-loud according to the $q_{12}$ parameter, defined as $q_{12}=\log \left(S_{12 \mu \mathrm{m}} / S_{\text {radio }}\right)$, with $S_{12 \mu \mathrm{m}}$ and $S_{\text {radio }}$ being the flux densities at $12 \mu \mathrm{m}$ and in radio respectively (D'Abrusco et al. 2019).

KDEBLLACS lists 5580 candidate BZBs (hereinafter labelled as KBZBs). This number decreases to 2807 sources lying in the SDSS footprint, however only 402 of them have good (i.e., $S / N>5$ ) optical spectra. Our final sample consists of these 402 sources together with 4 sources listed in Roma-BZCAT which have low-significance $(S / N<5)$ SDSS spectra. This sample corresponds to $7 \%$ of the whole KDEBLLACS (see Fig. 2 for details).

\section{Spectral analysis and classification}

In order to classify sources in WIBRaLS2 and KDEBLLACS according to their optical spectra, we started by cross-matching both previously defined samples with Roma-BZCAT (Massaro et al. 2015b), finding a total of 409 counterparts (out of 1424) for WIBRaLS2 and 78 (out of 404) for KDEBLLACS, respectively. These sources have already been classified as blazars and we assumed their classification without further verification.

We then adopted the following classification scheme, divided into sources listed as Roma-BZCAT or "remaining sources": Roma-BZCAT: BZBs, BL Lacs galaxy-dominated (BZGs), blazars of uncertain type (BZU), BZQs; and remaining sources: $\mathrm{BZB}$, quasi-stellar object (QSO) or BZQ in some cases, lowluminosity active galactic nuclei (LLAGNs), star-forming galaxies, BZGs, galaxies, and stars. Figure 3 shows the typical smoothed spectra for all these classes. The criteria adopted in the classification of the new analyzed sources is explained below. Particularly for blazars, we classified sources as BZBs when they showed a featureless optical spectra with a dominant blue continuum, and as QSOs when presenting redshifted broad emission lines again above a dominant blue continuum coupled with a radio luminosity $L_{\mathrm{r}}$ greater than $10^{38} \mathrm{erg} \mathrm{s}^{-1}$.

On the other hand, LLAGNs and star-forming galaxies were distinguished based on their line ratios $[\mathrm{OIII}] / \mathrm{H}_{\beta}$ and $[\mathrm{NII}] / \mathrm{H}_{\alpha}$ according to the BPT diagram (Baldwin et al. 1981; Kewley et al. 2006). A total of four star-forming galaxies were identified in WIBRaLS2 and only two in KDEBLLACS.

To distinguish between normal galaxies and BZGs, we measured their relative flux depression bluewards of the Ca II break. This parameter was defined as $\mathrm{CB}=\left(F_{+}-F_{-}\right) / F_{+}$, with $F_{+}$ and $F_{-}$meaning the flux densities measured in ranges of $200 \AA$ at wavelengths just above and below that of the $\mathrm{Ca}$ II $\mathrm{H}$ and $\mathrm{K}$ break (Dressler \& Shectman 1987; Massaro et al. 2012b). As in Stocke et al. (1991), we adopted a threshold value of 0.25 for $\mathrm{CB}$ to ensure the presence of a substantial nonthermal continuum (i.e., $\mathrm{CB}<0.25$ ) leading to a BZG classification; otherwise, the object in question was classified as a normal galaxy. Finally, a total of three and two stars were found contaminating WIBRaLS2 and KDEBLLACS respectively; four of them typical cold M- or G-type, and one white dwarf.

\section{Results}

The optical classification previously described enabled us to characterize WIBRaLS2 and KDEBLLACS subsamples. The results are presented here, split into the following subsections.

\subsection{WIBRALS2}

We analyzed a total of 1424 spectra (1396 with $S / N>5$ and 28 with $S / N<5$ but with a counterpart in Roma-BZCAT) among the 9541 sources available in WIBRaLS2 - but only 3407 in the SDSS footprint. According to their WIBRaLS2 classification, these sources were divided into 471 WBZBs, 833 WBZQs, and 120 WMIXEDs. As can be seen in Fig. 4, the main contaminant class is QSOs. A significant fraction of these QSOs, however, may have a radio flat spectrum, indicating that they could be indeed blazars of BZQ type. Subsequently, we found that 

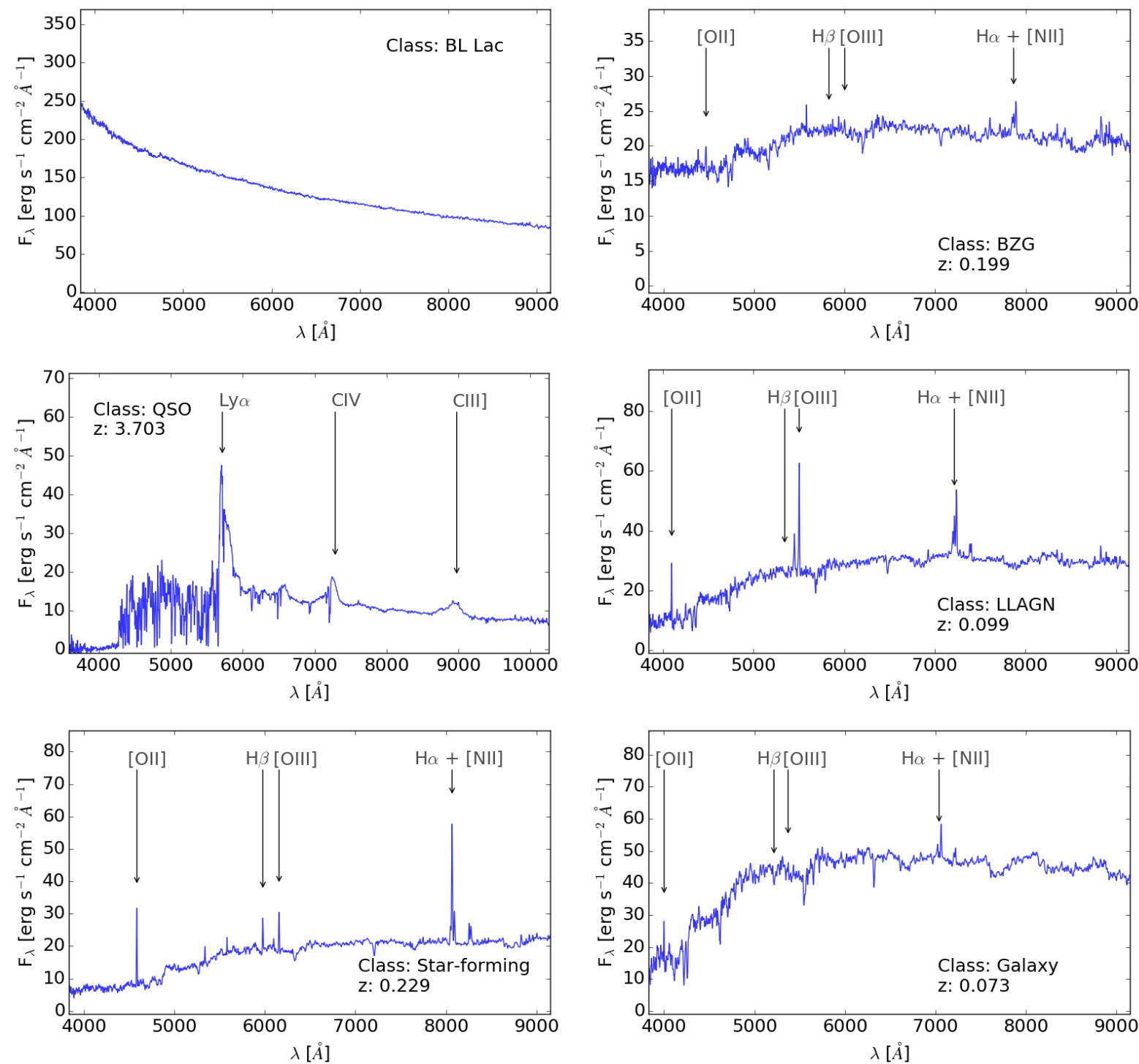

Fig. 3. Typical smoothed spectra in observed wavelengths for each one of the spectral categories found in WIBRaLS2 and KDEBLLACS.
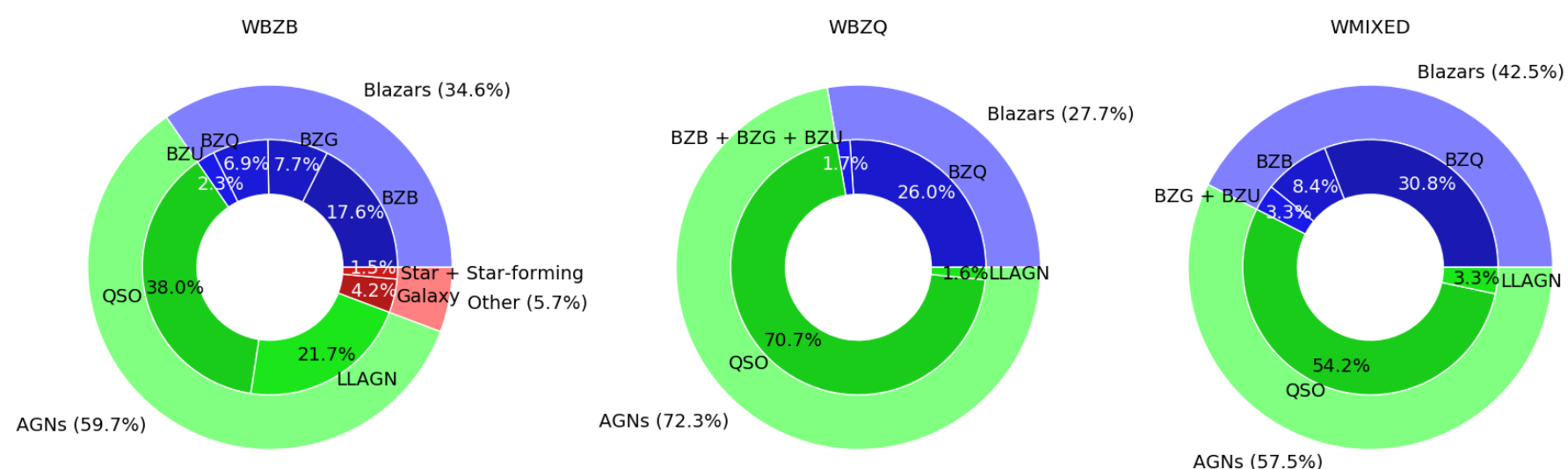

Fig. 4. Nested pie charts for the three classes of sources described in WIBRaLS2 (WBZB, WBZQ, and WMIXED). The major contaminants in all categories are AGNs. A significant fraction of these AGNs may however be blazars (BZQ). The subclass LLAGN is represented by Seyferts and LINERs.

$17.6 \%$ of WBZBs show a featureless optical spectrum; $26.1 \%$ of WBZQs are confirmed BZQs (i.e., radio flat spectrum) and $30.8 \%$ of WMIXEDs sources are also BZQs.
As can be seen in the left and middle panels of Fig. 5, the majority of contaminants in WIBRaLS2 are concentrated in the WBZQ and WMIXED regions of the MIR color-color diagram. 

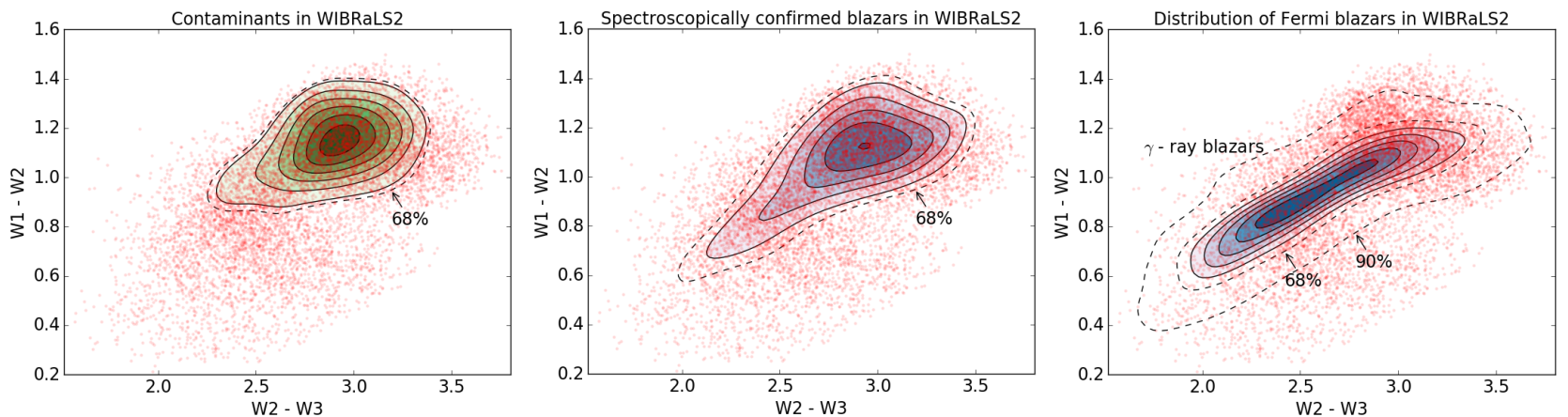

Fig. 5. Contaminants in WIBRaLS2. Left: contamination of WIBRaLS2 obtained based on a KDE and using the SDSS DR15 spectra of 953 sources (833 QSOs, 100 Seyferts and 20 LINERs) as a training sample. The contaminants are mainly QSOs concentrated in the WBZQ region of the color-color diagram (as pointed in Fig. 1). Middle: PDF of spectroscopically confirmed blazars in WIBRaLS2 (KDE training set composed of 281 BZQs, 95 BZBs, 36 BZGs and 24 BZUs). Most of them lie in the most contaminated region of WIBRaLS2. Right: PDF of $\gamma$-ray blazars obtained using 1320 cross-matches between 4FGL blazars and WIBRaLS2 sources as a training sample for a KDE. The $\gamma$-ray blazars are mainly located in a narrow strip peaking in the WBZB region. The solid lines represent the isodensity contours obtained with the KDE, while the dashed lines indicate the $68 \%$ and (for the last panel) $90 \%$.

On the other hand, the WBZB sample is cleaner, and its purity can reach $\sim 50 \%$ in the area bounded by the $68 \%$ isodensity contours of 4FGL $\gamma$-ray blazars (Fig. 5, right panel).

\subsection{KDEBLLACS}

With a total of 406 spectra analyzed, KBZBs in KDEBLLACS were classified as follows: 75 BZBs (60 of them confirmed in Roma-BZCAT), 39 BZGs (17 confirmed in Roma-BZCAT), 155 normal galaxies, 47 LLAGNs (20 LINERs and 27 Seyferts), 84 QSOs, 2 confirmed BZQs (1 of them listed in Roma-BZCAT), 2 stars, and 2 star-forming galaxies, all according to the criteria previously described. Figure 6 summarizes these results in a nested pie chart.

It is worth noting that normal galaxies and QSOs are the major contaminant classes of KDEBLLACS selection criteria. After applying two KDEs to this sample, one using spectroscopically confirmed QSOs and the other using spectroscopically confirmed galaxies as training sets, we observe (as expected) that these contaminants are concentrated towards the edges of the MIR color-color diagram (Fig. 7, left panel), mainly in the bottom and upper-right corners. Indeed, most of the BZBs classified in 4FGL lie outside of these regions (Fig. 7, right panel).

\subsection{Selection effects}

Since $\sim 52 \%$ of WIBRaLS 2 and $\sim 75 \%$ of KDEBLLACS sources in the footprint of SDSS have no available spectrum, we investigated possible selection effects, starting by comparing the typical optical colors of sources in WIBRaLS2 and KDEBLLACS with the colors of sources spectroscopically observed by SDSS, split by their SDSS spectroscopic classification. The lines in Fig. 8 show the $90 \%$ and $50 \%$ isodensity contours for 10000 spectroscopically classified galaxies (red), 10000 QSOs (green), and 10000 stars (black), randomly selected from SDSS DR15. Overall, the candidates from WIBRaLS2 and KDEBLLACS catalog occupy a very similar region of the $u-g$ versus $g-r$ optical diagram (Fig. 8). Most of the WIBRaLS2 and KDEBLLACS candidates are located within the $90 \%$ isodensity contours defined by spectroscopic SDSS QSOs and galaxies, which significantly overlap with the $90 \%$ contour for stars. In particular, in the case of WIBRaLS2 candidates (top panel), a large fraction (48\%) of candidates lie within the $50 \%$ contour of sources classified as QSOs, although other sources are scattered in the galaxy and

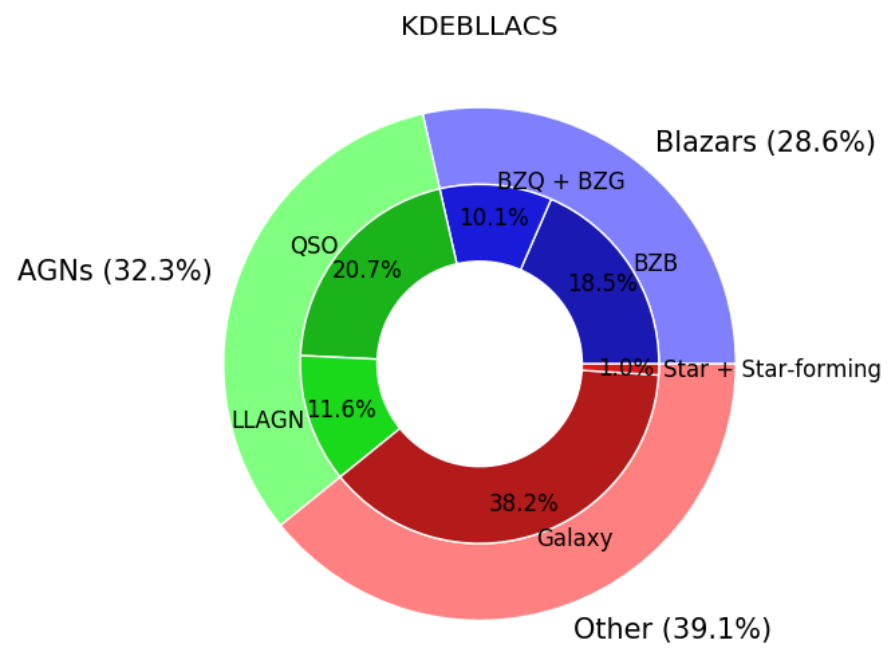

Fig. 6. Contaminants for KDEBLLACS divided by classes. The major contaminants of this catalog are normal galaxies. Some fraction of the QSOs could have a flat radio spectrum and be BZQs.

star-dominated areas. WIBRaLS2 candidates classified as WBZQ unsurpisingly peak in the area occupied by spectroscopically confirmed SDSS QSOs, while intermediate (WMIXED) and WBZB sources are spread over a much larger area consistent with both SDSS spectroscopic stars and galaxies. KDEBLLACS candidates are more evenly scattered through the isodensity contours for all three spectroscopic classes, likely indicating greater contamination from the host galaxies. Similar behaviors are visible in the other SDSS color-color diagrams, not reported in Fig. 8. In general, we can rule out the existence of significant selection effects due to the colors of the optical counterparts of our candidates.

Selection effects caused by the magnitude distribution of our candidates however are of major importance. As shown in Fig. 9, the large majority of the candidates missing optical spectra, especially for the KDEBLLACS catalog, are fainter than the candidates that have been followed-up spectroscopically. In particular, the reason why KDEBLLACS is spectroscopically less complete than WIBRaLS2 is because the former catalog has, in general, fainter sources. This effect is expected as KDEBLLACS candidates appear to be intrinsically fainter than WIBRaLS2 sources in all three WISE bands (W1,W2 and W3) where they are detected, as shown in Fig. 7 of D'Abrusco et al. (2019). 

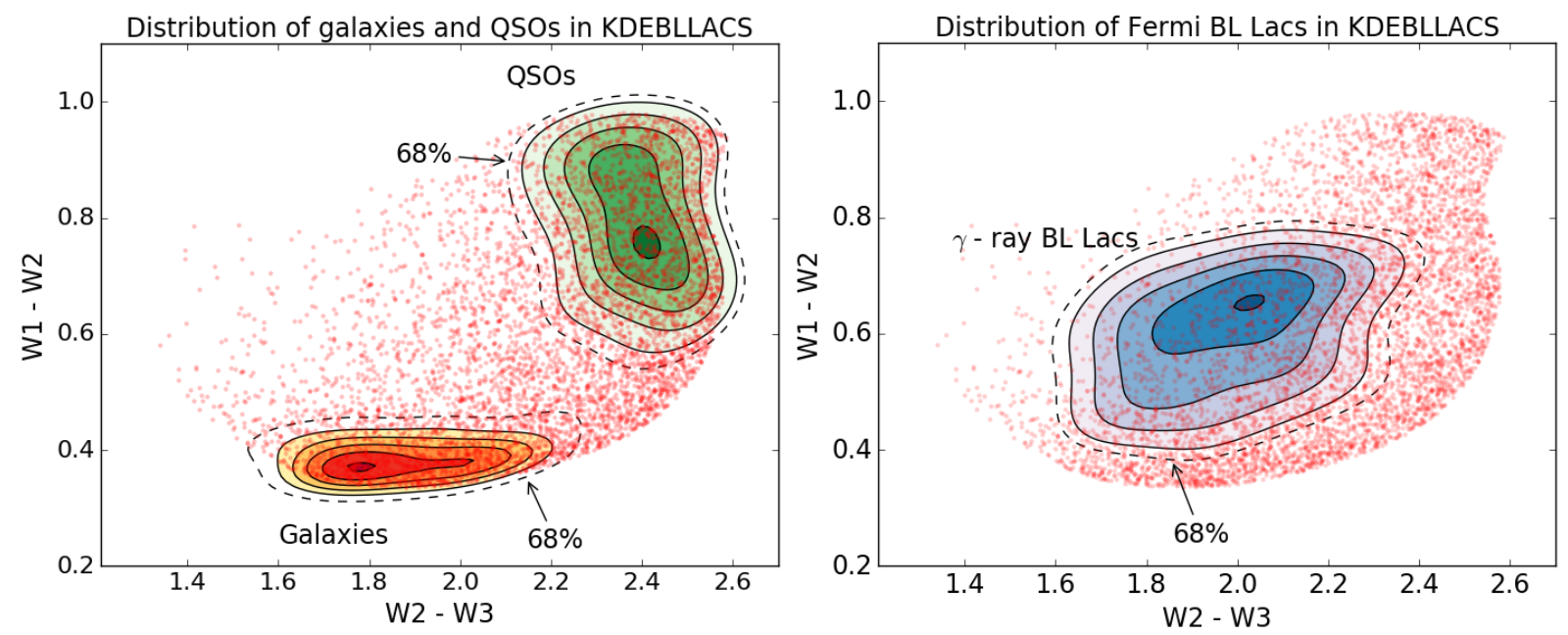

Fig. 7. Contamination of KDEBLLACS. Left: main contaminants of KDEBLLACS are normal galaxies and QSOs, both located at the edges of the sample. Right: PDF of the $\gamma$-ray BZBs available in 4FGL obtained with a KDE. We can see that the majority of BZBs are indeed located outside of the two contaminated zones. The solid lines represent the KDE isodensity contours, while the dashed lines represents the $68 \%$ isodensity contour.
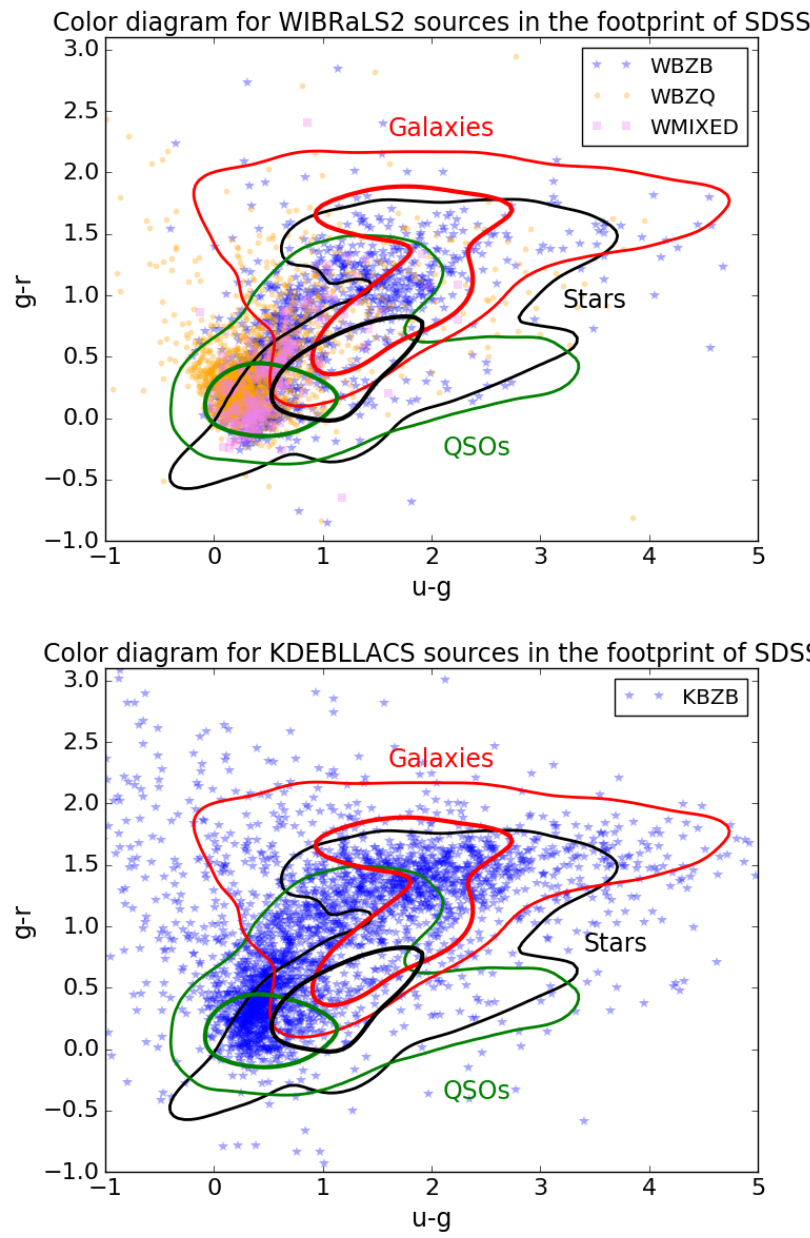

Fig. 8. Optical color-color diagrams for WIBRaLS2 (upper panel) and KDEBLLACS (bottom panel) sources in the footprint of SDSS. The total number of sources is 3407 for WIBRaLS2 and 2807 for KDEBLLACS, and are divided in WBZBs/KBZBs (blue stars), WBZQs (orange circles) and WMIXEDs (violet squares), according to their classification in WIBRaLS2 and KDEBLLACS. The red, green, and black lines represent, respectively, the $90 \%$ (thin line) and 50\% (thick line) isodensity contours based on the distribution of 10000 galaxies, 10000 QSOs, and 10000 stars with available spectra in SDSS DR15.
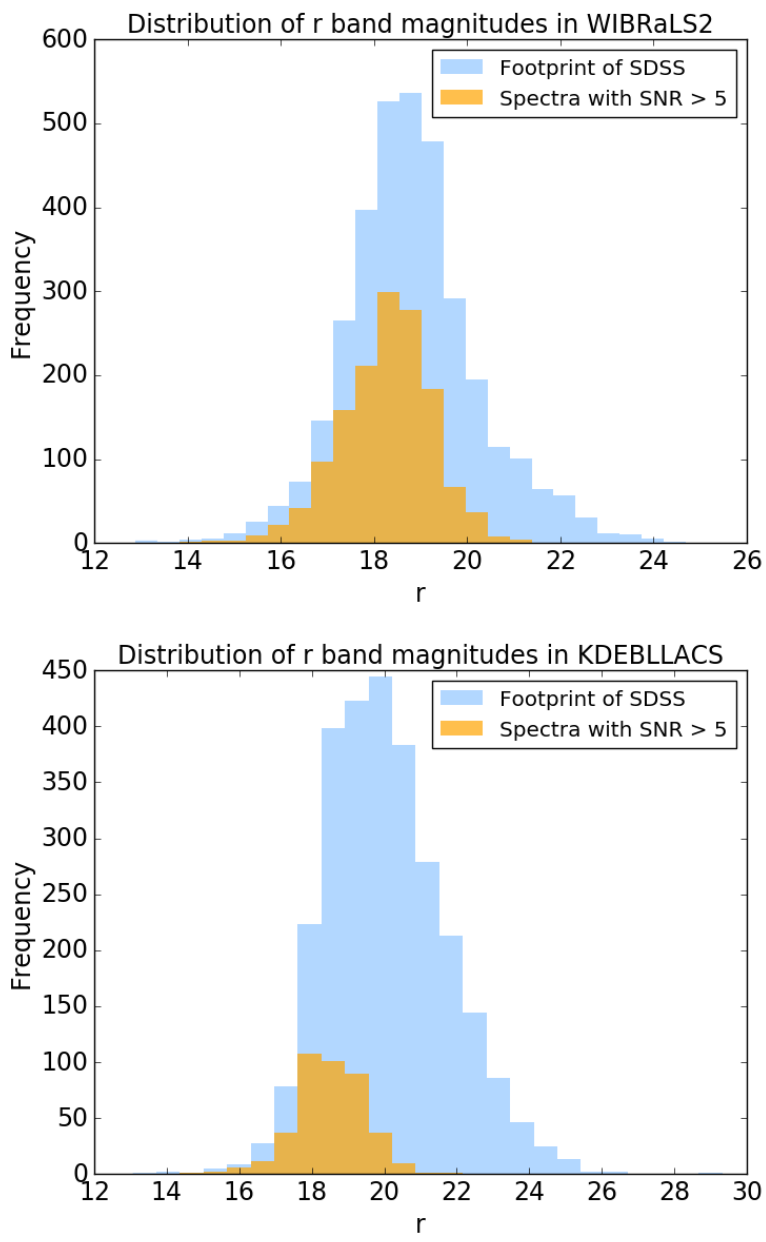

Fig. 9. Distribution of $\mathrm{r}$ band magnitudes for WIBRaLS2 (upper panel) and KDEBLLACS (bottom panel). The blue histograms represent all WIBRaLS2 and KDEBLLACS sources in the footprint of SDSS (3407 and 2807, respectively), while the orange histograms show only sources with high-quality $(S / N>5)$ spectra in SDSS DR15.

The magnitude distributions of WIBRaLS2 candidates with and without SDSS spectroscopic counterparts (upper panel in Fig. 9) are very similar. This suggests that other selection effects, likely 
Table 1. Spectral classification for 11 sources classified as BCUs in 4FGL.

\begin{tabular}{lccc}
\hline \hline WISE name & 4FGL name & Class & $z$ \\
\hline J003719.15+261312.6 & J0037.9+2612 & BZG & 0.1477 \\
J003820.53-020740.5 & J0038.7-0204 & RDG & 0.2204 \\
J013859.14+260015.7 & J0139.0+2601 & BZB & - \\
J020239.94-030207.9 & J0202.6-0258 & BZQ & 1.3444 \\
J084734.29+460928.0 & J0846.9+4608 & BZQ & 1.2165 \\
J094452.09+520233.4 & J0945.2+5200 & BZQ & 0.5630 \\
J095608.57+393515.8 & J0956.0+3936 & BZQ & 1.1730 \\
J130407.31+370908.1 & J1304.0+3704 & BZB & - \\
J134243.61+050432.1 & J1342.7+0505 & BZG & 0.1365 \\
\hline J123124.08+371102.2 & J1230.9+3711 & BZG & 0.2180 \\
J154150.09+141437.6 & J1541.7+1413 & BZG & 0.2230 \\
\hline
\end{tabular}

Notes. This table is segmented in WIBRaLS2 (upper part) and KDEBLLACS (bottom). The columns give the name of the sources as in WISE and 4FGL, our optical classification, and the redshift as in SDSS DR15.

based on the colors, extension, and morphology of the sources, increase the number of WIBRaLS2 sources lacking SDSS spectroscopic counterparts ${ }^{3}$.

\subsection{Blazar candidates of uncertain type}

Some sources in the Fermi-LAT catalogs are considered blazar candidates of uncertain type (BCUs) because the adopted association methods select a counterpart that satisfies at least one of the following conditions (Ackermann et al. 2015; Fermi-LAT Collaboration 2019): (i) An object classified as a blazar of uncertain or transitional type in Roma-BZCAT; (ii) a source with multiwavelength data indicating a typical two-humped blazar-like spectral energy distribution (SED) and/or a flat radio spectrum. The above-mentioned BCUs are divided into three sub-types (Ackermann et al. 2015):

- BCU I: the counterpart has a published optical spectrum that is not sensitive enough for its classification as BZQ or BZB.

- BCU II: there is no available optical spectrum but an evaluation of the SED synchrotron peak position is possible.

- BCUIII: the counterpart shows typical blazar broadband emission and a flat radio spectrum, but lacks an optical spectrum and reliable measurement of the synchrotron peak position.

In 4FGL, 1155 sources are considered to be BCUs. Our analysis based on the optical spectra available in SDSS DR15 (Sect. 3) allowed us to give a conclusive classification for 11 of them, as shown in Table 1.

The 4FGL catalog associated the source 4FGL J0038.70204 with the broad-line radio galaxy (RDG) 3C 17 at redshift $\sim 0.22$ as previously determined by Schmidt (1965) in agreement with the SDSS spectrum we analyzed. The kiloparsec-scale radio morphology of $3 \mathrm{C} 17$ is dominated by a single-sided, curved jet (Morganti et al. 1993) also shining in the X-rays (Massaro et al. 2009, 2010). Recent optical spectroscopic observations also revealed that $3 \mathrm{C} 17$ is the brightest cluster galaxy in its large-scale environment (Madrid et al. 2018).

\subsection{New BL Lacs}

During the spectroscopic classification (Sect. 3), we found a total of 25 (10 in WIBRaLS2 and 15 in KDEBLLACS) sources

\footnotetext{
3 For a general picture of the SDSS selection criteria for spectroscopic observations, see https://www.sdss.org/dr15/algorithms/ legacy_target_selection/
}

Table 2. New BZBs found during the spectroscopic classification in Sect. 3.

\begin{tabular}{lcc}
\hline \hline WISE name & SDSS name & $u-r$ \\
\hline J013859.14+260015.7 & J013859.14+260015.7 & 1.09 \\
J085446.24-003348.1 & J085446.22-003349.5 & 0.82 \\
J093522.08+502932.2 & J093522.08+502932.2 & 0.88 \\
J115406.13+185723.6 & J115406.11+185723.6 & 1.16 \\
J130407.31+370908.1 & J130407.32+370908.1 & 1.11 \\
J144906.05+071701.2 & J144906.04+071701.3 & 0.86 \\
J165558.59+391218.1 & J165558.61+391218.2 & 0.91 \\
J215601.64+181837.1 & J215601.64+181837.0 & 0.45 \\
J220812.70+035304.5 & J220812.70+035304.6 & 0.85 \\
J235915.62+221450.0 & J235915.62+221450.1 & 1.38 \\
\hline J000710.65-032029.4 & J000710.65-032029.4 & 0.74 \\
J020303.61-024547.3 & J020303.62-024546.9 & 0.82 \\
J024024.36-025334.3 & J024024.37-025334.3 & 0.70 \\
J083706.00+583152.9 & J083706.01+583152.9 & 0.60 \\
J083955.10+121702.9 & J083955.09+121703.0 & 0.71 \\
J091804.13+071653.6 & J091804.13+071653.6 & 1.22 \\
J101147.54+360018.7 & J101147.50+360018.8 & 0.68 \\
J103852.20+325651.7 & J103852.17+325651.6 & 1.22 \\
J111356.24+552255.3 & J11356.30+552255.6 & 0.60 \\
J114221.77+334201.8 & J114221.76+334201.8 & 0.85 \\
J114352.67+155821.9 & J114352.66+155822.0 & 0.51 \\
J135154.45+285008.0 & J135154.45+285007.9 & 1.26 \\
J154714.91+265442.2 & J154714.91+265442.4 & 0.77 \\
J171747.84+392607.3 & J171747.85+392607.3 & 0.99 \\
J171841.42+360522.6 & J171841.44+360522.2 & 1.32 \\
\hline
\end{tabular}

Notes. The first and second columns present the name of the objects in WISE and SDSS. The last column shows the color index $u-r$ based on SDSS AB magnitudes. The upper section of the table shows the BZBs found in WIBRaLS2 while the bottom section shows the ones found in KDEBLLACS.

with featureless optical spectra and a relatively strong blue continuum - typical characteristics of BZB nonthermal emission - which are not available in Roma-BZCAT. Their smoothed spectra, names and positions, and $r$ band magnitudes can be found in the Appendix A.

To check these new BZBs, we considered the $u-r$ color index of the SDSS photometric system (AB magnitudes), which is an efficient discriminator between BZBs and nuclei with weak or even absent activity (Massaro et al. 2012b). When computing these indexes, we considered the extinction-corrected SDSS model magnitudes according to the following formula:

$(u-r)=(u-r)_{\mathrm{obs}}-0.81 A_{r}$,

where $A_{r}$ is the extinction in $r$ band given in the SDSS database. Typically, BZBs present a color index $u-r \leq 1.4$ (Massaro et al. $2012 b$ ), however this criterium is not suited for sources at high redshifts $(z>0.5)$. Table 2 summarizes our findings.

\section{Summary and conclusions}

Here we characterized the two newly released catalogs of blazar candidates WIBRaLS2 and KDEBLLACS based on 1830 optical spectra -1798 spectra with $S / N>5$ and 32 with $S / N<5$ but with a counterpart in Roma-BZCAT - available in SDSS DR15 data. Both catalogs indeed presented a high number of spectroscopically confirmed blazars, although the contamination level of WIBRaLS2 was $\sim 60 \%$, mainly due to QSOs, and the contamination of KDEBLLACS was $\sim 70 \%$, mainly due to QSOs and normal galaxies. We stress that these contamination estimates represent upper limits due to the selection effects impacting the 
SDSS spectroscopic sample used in this paper, as described in Sect. 4.3. Our main results can be summarized as follows:

- Including the objects in Roma-BZCAT, 34.6\%, 27.7\%, and $42.5 \%$ of the sources in WBZB, WBZQ, and WMIXED, respectively, are blazars, which gives an overall weighted lower limit to the efficiency of WIBRaLS2 blazar selection of $\approx 31 \%$, with the major contaminants being, as expected, QSOs.

- The lower limit to the efficiency of KDEBLLACS in identifying blazars is $\sim 30 \%$, and its contaminants - mainly galaxies and QSOs - are concentrated on the edges of the MIR color-color diagram (Fig. 7).

- The spectral analysis carried out in Sect. 3 led us to the discovery of 25 new BZBs not available anywhere else in the literature, and to the classification of 11 BCUs listed in 4FGL.

This work contributes to a better understanding of the $\gamma$-ray sky in the Fermi-LAT era. In particular, the community will benefit from the characterization of WIBRaLS2 and KDEBLLACS in population studies of blazars and in subsequent programs of spectroscopic follow-up needed to confirm the nature of the UGSs.

Acknowledgements. We thank the anonymous referee for constructive comments which helped to improve the manuscript. The accomplishment of this project was only possible due to the ongoing support from the São Paulo Research Foundation (FAPESP), grants 2016/25484-9, 2018/24801-6 and 2017/01461-2. PH acknowledges support from the CONACyT program for Ph.D. studies. FR acknowledges support from FONDECYT Postdoctorado 3180506 and CONICYT Chile grant Basal-CATA PFB-06/2007. R.D'A. is supported by NASA contract NAS8-03060 (Chandra X-ray Center). This work is supported by the "Departments of Excellence 2018 - 2022" Grant awarded by the Italian Ministry of Education, University and Research (MIUR) (L. 232/2016). This research has made use of resources provided by the Compagnia di San Paolo for the grant awarded on the BLENV project (S1618_L1_MASF_01) and by the Ministry of Education, Universities and Research for the grant MASF_FFABR_17_01. F.M. acknowledges financial contribution from the agreement ASI-INAF n.2017 14-H.0. A.P. acknowledges financial support from the Consorzio Interuniversitario per la fisica Spaziale (CIFS) under the agreement related to the grant MASF_CONTR_FIN_18_02. TOPCAT8 (Taylor 2005) was extensively used in this work for the preparation and manipulation of the tabular data. This publication makes use of data products from the Wide-field Infrared Survey Explorer which is a joint project of the University of California, Los Angeles, and the Jet Propulsion Laboratory/California Institute of Technology, funded by the National Aeronautics and Space Administration. Funding for the Sloan Digital Sky Survey IV has been provided by the Alfred P. Sloan Foundation, the U.S. Department of Energy Office of Science, and the Participating Institutions. SDSS-IV acknowledges support and resources from the Center for HighPerformance Computing at the University of Utah. The SDSS web site is www.sdss.org. SDSS-IV is managed by the Astrophysical Research Consortium for the Participating Institutions of the SDSS Collaboration including the Brazilian Participation Group, the Carnegie Institution for Science, Carnegie Mellon University, the Chilean Participation Group, the French Participation Group, Harvard-Smithsonian Center for Astrophysics, Instituto de Astrofísica de Canarias, The Johns Hopkins University, Kavli Institute for the Physics and Mathematics of the Universe (IPMU)/University of Tokyo, the Korean Participation Group, Lawrence Berkeley National Laboratory, Leibniz Institut für Astrophysik Potsdam (AIP), Max-Planck-Institut für Astronomie (MPIA Heidelberg), Max-Planck-Institut für Astrophysik (MPA Garching), Max-Planck-Institut für Extraterrestrische Physik (MPE), National Astronomical Observatories of China, New Mexico State University, New York University, University of Notre Dame, Observatário Nacional/MCTI, The Ohio State University, Pennsylvania State University, Shanghai Astronomical Observatory, United Kingdom Participation Group, Universidad Nacional Autónoma de México, University of Arizona, University of Colorado Boulder, University of Oxford, University of Portsmouth, University of Utah, University of Virginia, University of Washington, University of Wisconsin, Vanderbilt University, and Yale University. The Fermi-LAT Collaboration acknowledges generous ongoing support from a number of agencies and institutes that have supported both the development and the operation of the LAT as well as scientific data analysis. These include the National Aeronautics and Space Administration and the Department of Energy in the United States, the Commissariat à l'Energie Atomique and the Centre National de la Recherche Scientifique/Institut National de Physique Nucléaire et de Physique des Particules in France, the Agenzia Spaziale Italiana and the Istituto Nazionale di Fisica Nucleare in Italy, the Ministry of Education, Culture, Sports, Science and Technology (MEXT), High Energy Accelerator Research Organization (KEK) and
Japan Aerospace Exploration Agency (JAXA) in Japan, and the K. A. Wallenberg Foundation, the Swedish Research Council and the Swedish National Space Board in Sweden. Additional support for science analysis during the operations phase is gratefully acknowledged from the Istituto Nazionale di Astrofisica in Italy and the Centre National d'Études Spatiales in France. This work performed in part under DOE Contract DE-AC02-76SF00515. This work is part of a project that has received funding from the European Union's Horizon 2020 Research and Innovation Programme under the Marie Skłodowska-Curie grant agreement NO 664931.

\section{References}

Abdo, A., Ackermann, M., Agudo, I., et al. 2010, ApJ, 716, 30 Acero, F., Ackermann, M., Ajello, M., et al. 2015, ApJS, 218, 23 Ackermann, M., Ajello, M., Atwood, W., et al. 2015, ApJ, 810, 14 Aguado, D., Ahumada, R., Almeida, A., et al. 2019, ApJS, 240, 23 Baldwin, J. A., Phillips, M. M., \& Terlevich, R. 1981, PASP, 93, 5

Blandford, R., Rees, M., \& Wolfe, A. 1978, Pittsburg Conference on BL Lac Objects (Pittburg: Pittsburg Univ. Press), 328

Böttcher, M. 2007, The Multi-Messenger Approach to High-Energy GammaRay Sources (Berlin: Springer), 95

Condon, J. J., Cotton, W., Greisen, E., et al. 1998, AJ, 115, 1693

Crespo, N. Á., Masetti, N., Ricci, F., et al. 2016a, AJ, 151, 32

Crespo, N. Á., Massaro, F., Milisavljevic, D., et al. 2016b, AJ, 151, 95

Cutri, R., Wright, E., Conrow, T., et al. 2013, Explanatory Supplement to the AllWISE Data Release Products

Cutri, R. M., Wright, E. L., Conrow, T., et al. 2014, VizieR Online Data Catalog: II $/ 328$

D’Abrusco, R., Massaro, F., Ajello, M., et al. 2012, ApJ, 748, 68

D'Abrusco, R., Massaro, F., Paggi, A., et al. 2014, ApJS, 215, 14

D'Abrusco, R., Crespo, N. Á., Massaro, F., et al. 2019, ApJS, 242, 4

Dressler, A., \& Shectman, S. A. 1987, AJ, 94, 899

Fermi-LAT Collaboration 2019, ArXiv e-prints [arXiv:1902 . 10045]

Helfand, D. J., White, R. L., \& Becker, R. H. 2015, ApJ, 801, 26

Kewley, L. J., Groves, B., Kauffmann, G., \& Heckman, T. 2006, MNRAS, 372, 961

Landoni, M., Falomo, R., Treves, A., \& Sbarufatti, B. 2014, A\&A, 570, A126

Landoni, M., Falomo, R., Treves, A., Scarpa, R., \& Payá, D. R. 2015a, AJ, 150, 181

Landoni, M., Massaro, F., Paggi, A., et al. 2015b, AJ, 149, 163

Madrid, J. P., Donzelli, C. J., Rodríguez-Ardila, A., et al. 2018, ApJS, 238, 31

Mainzer, A., Grav, T., Bauer, J., et al. 2011, ApJ, 743, 156

Marchesini, E., Peña-Herazo, H., Crespo, N. Á., et al. 2019, Astrophys. Space Sci., 364, 5

Massaro, F., Harris, D., Chiaberge, M., et al. 2009, ApJ, 696, 980

Massaro, F., Harris, D., Tremblay, G., et al. 2010, ApJ, 714, 589

Massaro, F., D’Abrusco, R., Ajello, M., Grindlay, J., \& Smith, H. A. 2011, ApJ,

740, L48

Massaro, F., D’Abrusco, R., Tosti, G., et al. 2012a, ApJ, 750, 138

Massaro, E., Nesci, R., \& Piranomonte, S. 2012b, MNRAS, 422, 2322

Massaro, F., Masetti, N., D’Abrusco, R., Paggi, A., \& Funk, S. 2014, AJ, 148, 66

Massaro, F., D’Abrusco, R., Landoni, M., et al. 2015a, ApJS, 217, 2

Massaro, E., Maselli, A., Leto, C., et al. 2015b, Astrophys. Space Sci., 357, 75

Massaro, F., Thompson, D. J., \& Ferrara, E. C. 2016a, A\&ARv, 24, 2

Massaro, F., Crespo, N. Á., D’Abrusco, R., et al. 2016b, Astrophys. Space Sci., 361,337

Mauch, T., Murphy, T., Buttery, H., et al. 2003, MNRAS, 342, 1117

Morganti, R., Killeen, N., \& Tadhunter, C. 1993, MNRAS, 263, 1023

Paggi, A., Milisavljevic, D., Masetti, N., et al. 2014, AJ, 147, 112

Peña-Herazo, H. A., Marchesini, E. J., Crespo, N. Á., et al. 2017, Astrophys. Space Sci., 362, 228

Peña-Herazo, H., Massaro, F., Chavushyan, V., et al. 2019, Astrophys. Space Sci., 364, 85

Ricci, F., Massaro, F., Landoni, M., et al. 2015, AJ, 149, 160

Schmidt, M. 1965, ApJ, 141, 1

Stickel, M., Padovani, P., Urry, C., Fried, J., \& Kühr, H. 1991, ApJ, 374, 431

Stocke, J. T., Morris, S. L., Gioia, I., et al. 1991, ApJS, 76, 813

Taylor, M. B. 2005, in Astronomical Data Analysis Software and Systems XIV, ASP Conf. Ser., 347, 29

Tegmark, M., Strauss, M. A., Blanton, M. R., et al. 2004, Phys. Rev. D, 69, 103501

Urry, C. M., \& Padovani, P. 1995, PASP, 107, 803

White, R. L., Becker, R. H., Helfand, D. J., \& Gregg, M. D. 1997, ApJ, 475, 479

Wright, E. L., Eisenhardt, P. R., Mainzer, A. K., et al. 2010, AJ, 140, 1868

York, D. G., Adelman, J., Anderson, Jr., J. E., et al. 2000, AJ, 120, 1579 


\section{Appendix A: New BZB candidates}

2014; Landoni et al. 2015b; Ricci et al. 2015; Crespo et al. 2016a,b; Massaro et al. 2016b; Peña-Herazo et al. 2017, 2019; During the spectral classification process described in Sect. 3, Marchesini et al. 2019). Their smoothed optical SDSS DR15 we found 25 new BZBs not available in Roma-BZCAT or during our follow-up spectroscopic campaign (see e.g., Paggi et al. spectra $(S / N>5)$ as well as their $r$ band magnitudes are available in Figs. A.1 and A.2.
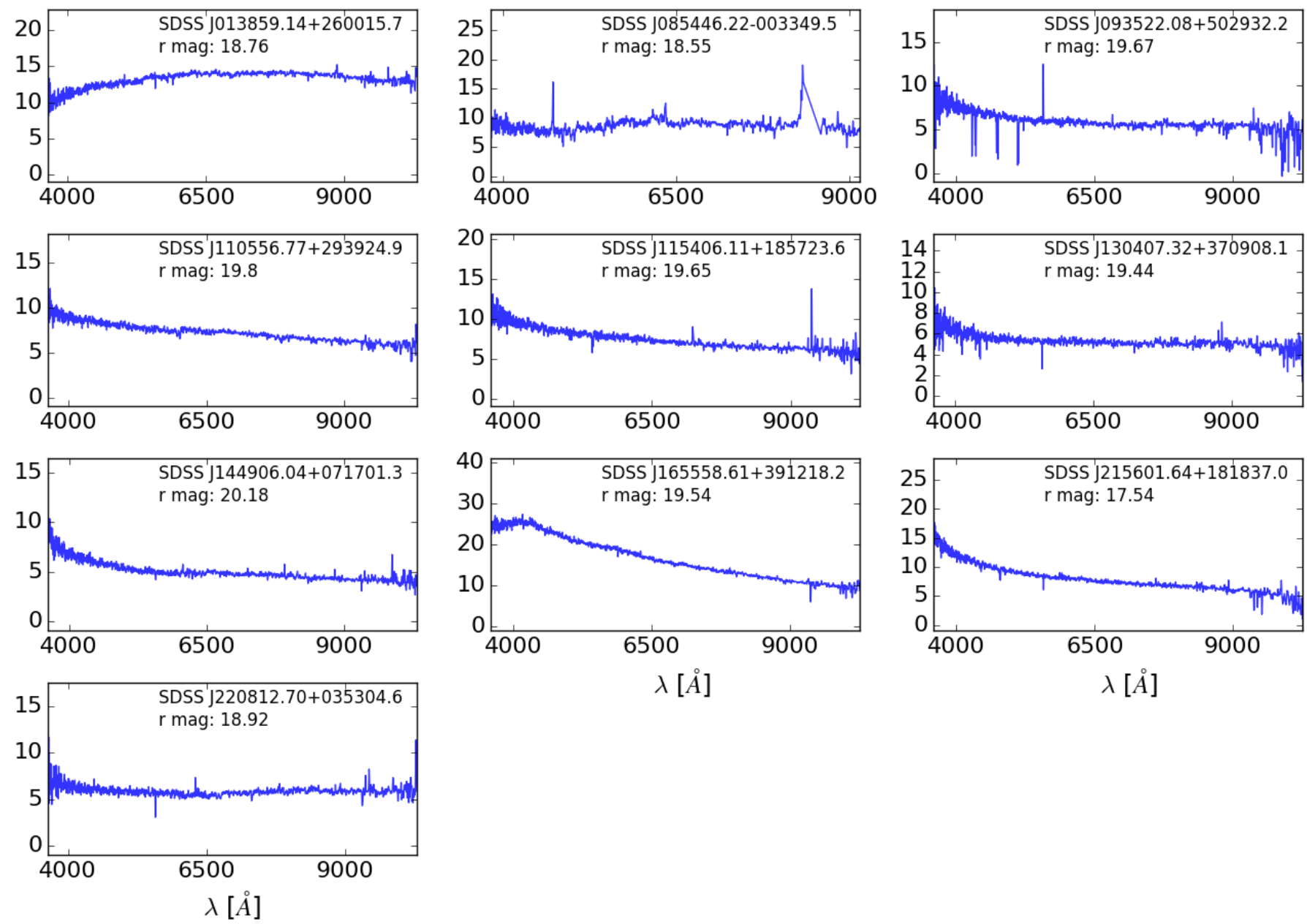

$\lambda[\AA]$

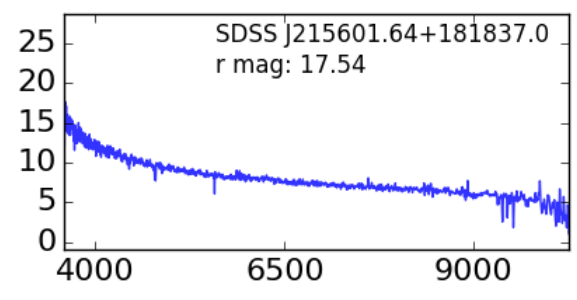

$\lambda[\AA]$

Fig. A.1. SDSS smoothed optical spectra of the new BZBs in WIBRaLS2. All sources present featureless spectra with color index $u-r<1.4$. We also show their SDSS name and $r$ magnitude. 

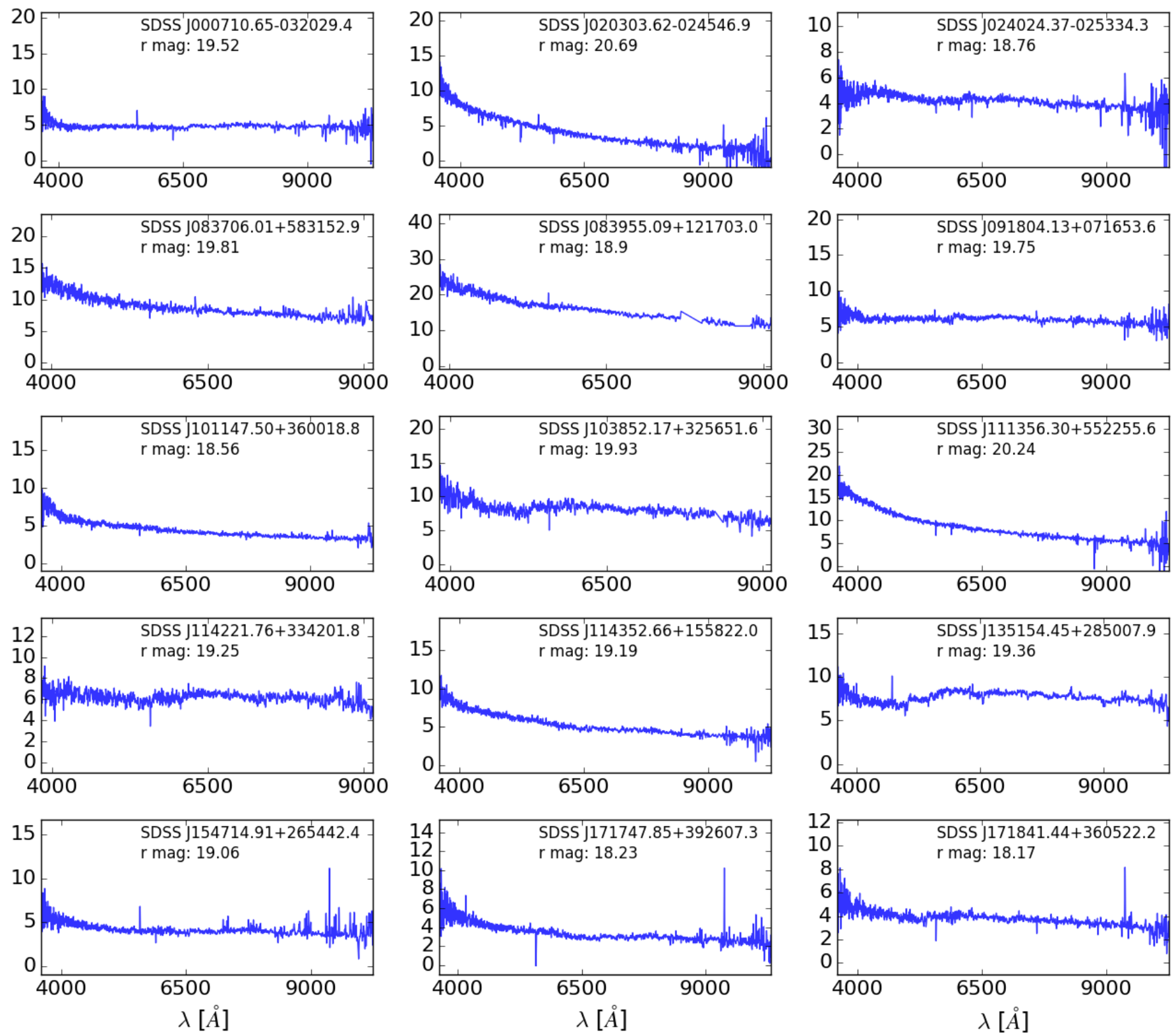

Fig. A.2. SDSS smoothed optical spectra of the new BZBs in KDEBLLACS. All sources present featureless spectra with color index $u-r<1.4$. We also show their SDSS name and $r$ magnitude. 